
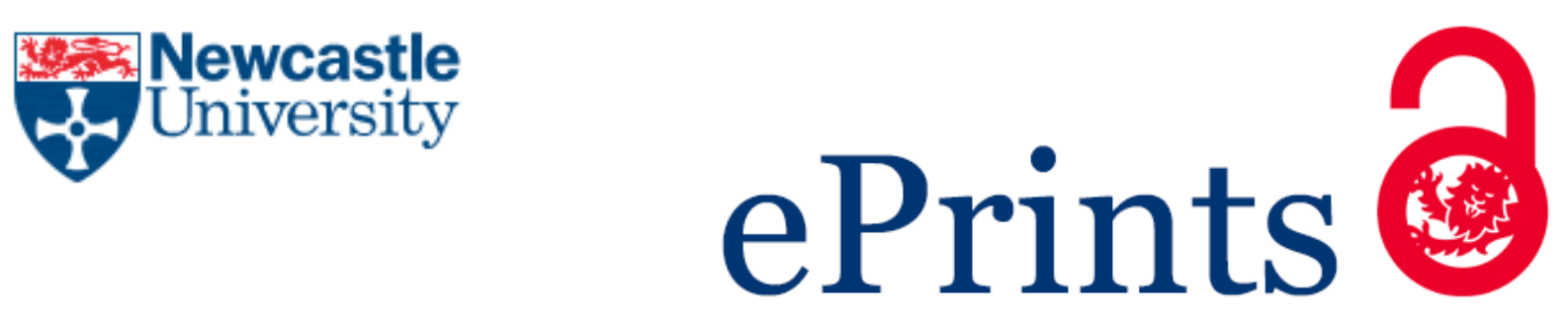

Lee RP, Hart RI, Watson RM, Rapley T.

Qualitative synthesis in practice: some pragmatics of meta-ethnography. Qualitative Research 2015, 15(3), 334-350.

\title{
Copyright:
}

This is the authors' accepted manuscript of an article that has been published in its final definitive form by Sage Publications, 2015.

Link to published article:

http://dx.doi.org/10.1177/1468794114524221

Date deposited:

$20 / 12 / 2015$

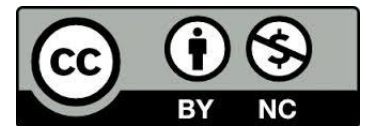

This work is licensed under a Creative Commons Attribution-NonCommercial 3.0 Unported License 


\section{Qualitative synthesis in practice: some pragmatics of meta-ethnography}

Richard Lee, Ruth Hart, Rose Watson, Tim Rapley

Institute of Health \& Society

Newcastle University

Baddiley-Clark Building

Richardson Road

Newcastle-upon-Tyne

NE2 4AX

\section{Abstract}

Synthesising qualitative research involves working through difficult practical issues. Drawing upon our collective experience of undertaking three meta-ethnographies, we consider the forms of work - the practical action and practical reasoning - comprising this kind of synthesis and the difference they make to a meta-ethnography. We detail the origins and aims of meta-ethnography, and present a review of existing meta-ethnographies with a specific focus on the methods the authors reported as central to the conduct of meta-ethnography. We consider the implications of these methods and the reason for the presence (and absence) of particular practices in reporting on meta-ethnographies. Drawing upon our own experiences of conducting meta-ethnographies we focus on the methods used in two key practices central to meta-ethnography: 'reading' and 'conceptual innovation'. We conclude by discussing how the 
meta-ethnographic process requires active reading, a recognition of multiplicity, a realistic approach to conceptual innovation and, importantly, collaborative work.

\section{Keywords}

meta-ethnography, qualitative research, research synthesis, review, methods, pragmatism, health services research 


\section{Introduction}

Over the last decade the diversity of approaches to synthesising qualitative research has grown steadily and has itself become a topic for review. Championed approaches include: meta-ethnography; qualitative meta-synthesis; realist review; thematic analysis; critical interpretive synthesis; and framework analysis/synthesis. It is evident that many, though not all, of these approaches to interpretive synthesis share a common history, methodologically rooted in the application and development of meta-ethnography (Noblit and Hare, 1988) and politically located in efforts to establish the value of qualitative research in the context of the rise of evidence-based policy and practice (Hammersley, 2013). However, as Hannis and Macaitis (2012) have suggested in this journal, detailed discussion of the practical methods used to achieve a qualitative synthesis is seldom a feature of published accounts.

In this paper we examine how meta-ethnography is conducted in practice, drawing upon our collective experience of undertaking three meta-ethnographies. While endeavouring to produce three separate syntheses of studies in particular domains - informal caring for musculoskeletal conditions, young people's involvement in treatment decision-making, health service transitions - we paid particular attention to the practical methods - the practical actions and practical reasoning - we employed to carry out each meta-ethnography and compared the unfolding and situated processes.

Our intention here is not to critique existing meta-ethnographies, but to consider the forms of work comprising this kind of synthesis and to consider the difference this might make to a metaethnography. First we detail the origins, aims and influence of meta-ethnography. We then present a 
brief review of existing meta-ethnographies, with a specific focus on the methods authors reported as central to conducting the meta-ethnography. We consider the implications of these methods and the reason for the presence (and absence) of particular activities in the reporting of meta-ethnographies. Having discussed how the conduct of meta-ethnography has been described in the literature, we draw upon our own experiences of conducting meta-ethnographies to consider the pragmatics of two key activities: 'reading' and 'conceptual innovation'. In recognising the challenges of producing a synthesis, we conclude by discussing the importance of collaborative work to address the implications of coherence and multiplicity for the meta-ethnographic process

\section{Meta-ethnography: its origins, aims and influence}

Meta-ethnography has occupied a prominent role in the conceptual and practical development of qualitative synthesis. In a recent overview, Paterson (2012) suggests that the delineation of metaethnography by Noblit and Hare (1988) gave rise to the 'first phase' of qualitative synthesis. She notes that meta-ethnography has formed the basis of many subsequent synthesis studies and has been adapted over time towards particular ends.

The authors of meta-ethnography, George Noblit and Dwight Hare, sought to articulate a method of synthesis which dealt with the interpretation of a small number of ethnographic studies based on available monographs. They proposed three trajectories of synthesis a meta-ethnography could follow:

- reciprocal translations as synthesis - when studies 'are about similar things' (Noblit and Hare 1988: 38), translate different accounts into one another;

- refutational synthesis - when studies 'refute each other' (ibid), interpret conflicting accounts; 
- line-of-argument synthesis - when studies 'successively "build" a line of argument' (ibid) develop an over-arching interpretation.

In terms of process, they delineated seven iterative phases of meta-ethnography and in doing so provided a procedure which has subsequently been utilised and adapted. In the following section we examine how different research teams have applied the method of meta-ethnography.

\section{Meta-ethnography on paper: A consideration of existing work}

Reflecting on the relative popularity of meta-ethnography as detailed in their work of 1988, George Noblit admits that he and his co-author '...thought the book had died a suffocating library death in the mid-1990s' (Thorne et al, 2004: 1347). A synthesis by Paterson et al (1998) and a review by Sandelowski et al (1997) represent early reappraisals of meta-ethnography, the former identifying and developing metaphors on the basis of 38 studies, the latter comparing meta-ethnography to other approaches to synthesis of qualitative studies. Neither article elaborates on the process and logic of meta-ethnography. In the following years further work directly influenced by, or responding to, metaethnography emerged, with examples including: Britten et al (2002); Rice (2002); Campbell et al (2003); Doyle (2003); Thorne et al (2004); Dixon-Woods et al (2005); Pound et al (2005); Dixon-Woods et al (2006); Atkins et al (2008); Flemming (2010); Schumm et al (2010); Campbell et al (2011); Hannes and Macaitis (2012); and Tondeur et al (2012).

Work influenced by meta-ethnography varies in how explicit authors are in reporting on the pragmatics of conducting meta-ethnography. Dixon-Woods et al (2005) and Campbell et al (2011) are 
perhaps the most detailed and sustained treatments addressing specific activities and methods. DixonWoods et al (2005) address the origins and content of meta-ethnography as set out by Noblit and Hare (1988) and go on to develop an approach they term 'critical interpretive synthesis'. They suggest that, in 2005, few worked applications of meta-ethnography were available, stating that:

Meta-ethnography as originally proposed, however, is solely a method for synthesis, and offers little guidance on how to formulate questions for review, how to identify, appraise, and select studies for inclusion in a review, or on the procedures for conducting a synthesis (Dixon-Woods et al, 2005: 14).

They also note the work of Britten, Campbell and colleagues who have sought to modify metaethnography in order to deal with relatively large numbers of studies. Indeed, this concern with the ability of meta-ethnography to deal with large numbers of potentially diverse studies in part spurs them to differentiate critical interpretive synthesis from meta-ethnography.

For Campbell et al (2011) meta-ethnography represents a form of interpretive synthesis which, as originally conceived, resisted making the process of data analysis explicit. They suggest that Noblit and Hare 'were critical of the emphasis given by some qualitative researchers to the explicitness of processes employed to analyse data' (Campbell et al, 2011: 11). Despite these concerns, the growth in the application of meta-ethnography as a form of synthesis means that potentially valuable lessons might be learnt from better understanding the practical actions and reasoning researchers employ in order to realise the methodology. 
Noblit and Hare's seven-step iterative process remains the primary organising device for conducting meta-ethnography. Teams reporting on a meta-ethnography often discuss how each step was addressed. Figure 1 outlines the activities, as reported in ten accounts of the meta-ethnographic process. These accounts were selected as they gave the reader technical access to the process of metaethnography. Other accounts were excluded due to insufficient detail about the process. Six of these accounts are applications of meta-ethnography to specific synthesis questions (Britten et al, 2002; Rice, 2002; Campbell et al, 2003; Pound et al, 2005; Schumm et al, 2010; Tondeur et al, 2012), one is a consideration of meta-ethnography (Doyle, 2003), two discuss meta-ethnography alongside other synthesis approaches (Thorne et al, 2004; and Dixon-Woods et al, 2005) and one applies a synthesis approach (critical interpretive synthesis) related to meta-ethnography (Dixon-Woods et al, 2006). Using the seven-step process as our framework, along with guidance from Noblit and Hare themselves (shown in bold), we present selected material from these papers ${ }^{1}$ to demonstrate the different ways in which the steps have been operationalized. For reasons of space, we focus on activities associated by the authors of the reviewed papers with steps 3-6 - steps which, as we explain below, present considerable opacity and challenge.

\footnotetext{
${ }^{1}$ Short references are used: N\&H: Noblit and Hare (1988); Brit: Britten et al (2002); Rice: Rice (2002); Cmbl: Campbell et al (2003; 2011); Doyle: Doyle (2003); Dix: Dixon-Woods et al (2005); Pnd: Pound et al (2005); Atk: Atkins et al (2008); Schm: Schumm et al (2010); Tond: Tondeur et al (2012).
} 
1.GETTING STARTED

\section{DECIDING WHAT'S RELEVANT}

3.READING THE STUDIES - Read in detail and identify metaphors for each case and assess adequacy of metaphors (N\&H)

- Document information on context (Brit)

- Organise studies into groups (Pnd)

- $\quad$ Code themes; another researcher checks coding (Rice)

4. DETERMINING HOW RELATED - List and juxtapose metaphors for each case and decide how studies relate and type of synthesis to apply (N\&H)

- $\quad$ Code themes; identify categories (Atk) (Dix)

- $\quad$ Find common and recurring concepts (Brit)

- Record contextual information, concepts, and key theory from each paper (Brit)

- List summary of findings, using original terms and concepts (Cmbl)

- Identify 'key concepts' and relationships between them (Cmbl)

- Map key findings within groups of studies (Pnd)

- Compare themes while reading each study (Rice)

- Another researcher checks coding (Schm)

5. TRANSLATING THE STUDIES - Construct translations, preserving within-study relationships between metaphors whilst comparing across studies (N\&H)

- Review each paper for presence/absence of concepts (Atk; Cmbl; Schm; Tond)

- Derive concepts that encompass more than one study ( $\mathrm{Cmbl})$

- Consider similarities/differences (Rice)

- Develop a conceptual model across groups of studies (Pnd)

- Be reflexive (Doyle)

- Maintain language of original terms and concepts (Brit; Doyle)

- Create matrix, review adequacy of concepts for each case (Brit)

\section{SYNTHESISING THE TRANSLATIONS - Collect the translations and check if any translations or} metaphors can encompass others (N\&H)

- Review matrix to see relationships between concepts and studies (Brit)

- Use a key theory from each paper as 'building blocks' for the synthesis (Brit)

- Move from cases as part of collection to a final narrative (Doyle)

- Develop a synthesising argument, might require synthetic constructs (Dix)

- Produce concept(s) that explain all the data (Pnd)

- Produce models and then create line-of-argument synthesis (Atk)

- Use frameworks created from theory to categorise themes (Rice)

- Develop line-of-argument synthesis (Schm)

\section{EXPRESSING THE SYNTHESIS}

Figure 1: How a range of different authors describe doing meta-ethnography 
Initial scrutiny of Figure 1 suggests that a variety of ways to enact the 'steps' of metaethnography have been developed. For reasons of space, activities related to 'Getting started' and 'Deciding what's relevant' are not detailed in the version of Figure 1 presented here, but were found to move beyond Noblit and Hare by specifying techniques now relatively common to the procedural norms of synthesis in more quantitative styles, including the use of search term definitions, expert panels and quality appraisal tools to identify coherent and relevant studies.

Doyle (2003) discusses the application of boundary conditions for relevant cases in order to exclude purely descriptive accounts. The conceptual depth, or the thinness of categories, evident in studies, and the implications of this for conducting a meta-ethnography, will be discussed later in the paper. For now it is sufficient to note that explaining the search strategy used to identify studies in a meta-ethnography is much more straightforward than detailing the conduct of later steps and is, in part, a response to the demands for 'regulatory objectivity' (Cambrosio et al, 2006) from the evidence-based policy and practice movement.

While the identification of studies is an important element of meta-ethnography, detailing the work of reading, relating, translating, synthesising and expressing (steps 3-7) is arguably a more difficult, and perhaps more significant, task, particularly given the constraints of available space for publishing meta-ethnographies. As these activities are central to the pragmatics of this approach, it is necessary to review how the reporting of the practical conduct of steps 3-7 is achieved and to consider different enactments and potential silences. 
In most of the meta-ethnographies reviewed, 'Reading the studies' is the phase in which the accounts begin to be considered in terms of data to be extracted, coded and interpreted. For some, this step reveals the largely descriptive (or 'thin') content of studies (Atkins et al, 2008). For others, grouping the studies was a reading activity (Campbell et al, 2003; Pound et al, 2005), giving rise to anxiety: 'At the outset, it was not clear whether these papers could be synthesised' (Campbell et al, 2003: 674). However, less detail is provided in published meta-ethnographies on reading than on later phases. Reading, as we will discuss later in the paper, comprises a set of activities which we argue permeate the meta-ethnographic process and this may go some way to explain why there is little discussion of reading as a distinct phase.

Determining relations between studies, translating the studies and synthesising the translations represent the bulk of the labour undertaken during the meta-ethnographic process. Our review (see Figure 1) suggests that relational and translation activities were undertaken in an iterative manner, with divergent approaches to the question of maintaining the differences between studies, as Noblit and Hare (1988) proposed and Noblit more recently reiterated (Thorne et al, 2004). In Pound et al's (2005) study of lay experiences of medicine taking, they discuss different steps in the process - initially grouping accounts by the medicines they related to and seeking to translate accounts within these subgroups. They then produced diagrams or 'maps' of the findings for each group and used these maps to draw together the findings from the different medicine groups:

'We determined how the findings related to each other across the medicine groups by comparing the medicine maps with each other and developing a model that was able to encompass the findings from all the studies in the synthesis' (Pound et al, 2005: 135). 
For Schumm et al (2010) moving through relational and translation activities involved producing firstorder (reported data) and second order (interpretations in the original studies) constructs. As in some other meta-ethnographies they report the development of 'third-order constructs' which 'were discussed and re-worked until team consensus was reached' (Schumm et al, 2010: 338).

One issue we wish to outline is the lack of detail often given to the methods undertaken to carry out relating and translating. There is a consensus that translation does not involve secondary analysis of data, and that 'the data of synthesis are interpretations and explanations rather than the data collected through interviews and observations' (Noblit and Hare, 1988: 32). However, variation is evident in the efforts to retain the original text (emphasised by Doyle, 2003; less apparent in Britten et al, 2002). The movement from determining relationships to conducting a translation (and synthesis) of studies is not strictly linear. Campbell et al (2003) move from lists of findings and interrelated concepts within studies to their systematic interrogation and the development of concepts functioning across multiple studies. Atkins et al (2008) engage in an analysis of themes, produce categories, and then move to translate these categories across papers (in chronological order). Several studies (e.g. Schumm et al, 2010) refer to members of the research team conducting activities in parallel, for verification purposes.

It is seldom clear what activities have been (or should be) undertaken in order to arrive at a final synthesis - Britten et al, 2002, being an exception here. Perhaps this is inevitable, in that the creative and imaginative nature of this work acts against detailed or rigid specification. For Campbell et al (2011), meta-ethnography, as originally conceived, involves actively resisting the explicit statement of analytical and synthesising processes. However, there is some consensus that done properly it is a 
painstaking process requiring 'considerable immersion in the individual studies' (Campbell et al, 2011: xi). The laboriousness of the approach perhaps explains why interest does not seem lasting and Hannes and Macaitis (2012) suggest that few authors have contributed more than one qualitative synthesis.

\section{Meta-ethnography in action: Learning from experience}

Previous meta-ethnographies have suggested that teamwork can be used to identify and assess literature, extract and cross-check material (data) and agree on coding schemes and interpretation. There is a focus here upon ensuring validity. In contrast to Atkins et al (2008), who detail lessons learnt from the conduct of a single meta-ethnography by a multi-disciplinary team, our collaborative approach involved making sense of the process through different meta-ethnographies being conducted by members of the team. Rather than working collectively to produce a single synthesis, we were interested in conducting three meta-ethnographies on different substantive topics, paying particular attention to the methodological issues raised within each meta-ethnography and making procedural comparisons across the meta-ethnographies. From the initial inception of the idea to the later stages of synthesis, we wrote methodological memos about the process and met regularly. We wrote field notes about our meetings, many of which were audio-recorded and the recordings transcribed. Our focus was on articulating and making sense of the hidden labour of meta-ethnography. 


\section{Hidden labour with an uncertain return}

The intellectual labour required to produce a meta-ethnography involves working with metaphors or concepts within and between accounts. As evidenced in Figure 1, a variety of activities can be undertaken to produce the synthesis and this gives rise to two key questions:

- which methods to use to make sense of the accounts in relation to one another;

- $\quad$ which methods to use to synthesise the accounts.

Undertaking a meta-ethnography involves experimenting with these methods and cannot be approached as a linear process. The experimental nature of meta-ethnography means that the work undertaken may not produce a direct advancement towards the final synthesis, but may be necessary to enable decisions about how best to proceed. While the stages devised by Noblit and Hare continue to provide a framework for doing a meta-ethnography, we feel time must be invested in ascertaining the most productive methods with which to interpret and translate the accounts. Issues of labour and intellectual investment can benefit from a collaborative approach to meta-ethnography. As Barry et al (1999) have written, collaborative working not only improves quality and rigour, but subjects the analytical process to group reflexivity. For us, the explanation and debate inherent to collaborative working helped to address anxiety over the meta-ethnographic process.

As suggested above, the work involved in a meta-ethnography is seldom the subject of lengthy treatment. Given the demands to publish succinct summaries of the main products of a metaethnography, it is understandable that the practical process of doing meta-ethnography is often discussed in terms of search strategies, distribution of coding and analysis duties and the application of particular meta-ethnographic steps. What remains hidden is the labour involved in trying to apply a 
meta-ethnographic approach to qualitative studies, involving specific tasks which contribute to the final published version of the synthesis. In the remainder of the paper we draw upon our collective experiences of conducting three meta-ethnographies to discuss two key activities - 'reading' and 'conceptual innovation'. We consider the practical action and reasoning embedded in undertaking them and the implications for undertaking meta-ethnographies

\section{Reading(s) and meta-ethnography}

What emerged from our discussions was a growing conviction of the centrality of reading, as product and process, to both our individual and collective endeavours. At an individual level, we were seeking to produce a 'convincing reading' (Geertz, 1973) of the contributing accounts; effectively, through synthesis, arriving at a new 'take' on the phenomena. The work of our collective, arguably, was also to produce a new reading - this time of the practice of meta-ethnography - by drawing out and bringing together the lessons emerging from the application of its principles to three different areas and sets of accounts. However, it was the process and practices of reading - rather than the products of reading which most captured our attention.

As noted above, in writing for publication claims to credibility are often established through reference to recognised analytical practices, the detail of which (often for good reason) the audience is generally spared. However, the nature of our project required us to scrutinise and document closely the mundane practices underpinning our analyses and knowledge claims. Looking at our own work and that of others we identified more and more practices which might be grouped together as 'strategies for reading'. And notwithstanding the designation of the third meta-ethnographic step as 'Reading the 
studies' (Noblit and Hare, 1988), it began to seem obvious that reading pervaded the whole process. As $\mathrm{XW}^{2}$ commented in a pivotal meeting 'In some ways it's completely bizarre to have that as a particular phase, reading, because like you say that's what you do all the way through' (Meeting note, 26/07/2012). Though we later noted that Noblit and Hare (1988) identify reading as not easily 'confined' to one particular phase, and that repeated reading will be necessary, we had clearly not absorbed the significance of this point in our original reading of the monograph. Over time we came to believe that the intensive, repetitive, and above all highly active reading which all three meta-ethnographies involved was at the very heart of the meta-ethnographic process.

So what did such 'active reading' entail? It is clear from both our own efforts, and our review of the meta-ethnographies of others, that at different points in the process, reading might be undertaken with different intentions: to appraise; familiarise; identify; extract; record; organise; compare; relate; map; stimulate; and verify. A further array of techniques - annotating, coding, listing and tabulating might be employed to achieve these different purposes. Across the team we uncovered clear differences in approach, in particular around the sequencing and timing of individual techniques. For example, in her meta-ethnography of young people's involvement in treatment decision-making, $X X$ opted to identify and list 'metaphors' (key ideas, involving both 'in vivo' and paraphrased material), putting considerable emphasis on trying to capture the essence of individual accounts. In contrast, at a similar point in the process, XW started to develop a coding framework, using this to facilitate comparison of extracts from his chosen accounts.

\footnotetext{
${ }^{2}$ Initials refer to the authors: XW, Author $1 ; \mathrm{XX}$, Author 2; XY, Author 3; XZ, Author 4).
} 
In some respects such intra-team differences in approach mirror the variation we noted in the reviewed meta-ethnographies, with a lack of consensus as to how best to manage the shift in focus from working within to working across accounts. Where we as a collective were in agreement was that shifting - backwards and forwards - between reading within and across accounts is challenging. So whilst we were largely in accord that in the early stages of the process one 'should' read with an emphasis on the individual account, giving attention to its decisive metaphors and relationships (i.e. focus on 'reading within'), we all reported some level of difficulty in doing this. Echoing debates around the directives of first-generation grounded theory to avoid preconceptions from the literature (e.g. Dey, 1999), we talked recurrently and at some length about the struggle to bracket our knowledge of other accounts - those selected for inclusion and those rejected, in both cases decisions subject to review and our shared sense of the need, pragmatically, to read with the intention of collating a synthesisable set of accounts (i.e. to some extent always be reading across).

To one degree or another we were all taxed by the need to find ways to manage, and demonstrate management of, the essential tension between reading within and reading across. However, $X Y$ and $X X$ in particular harboured doubts about the suitability of accounts, and shared concerns about imminent difficulties. XX's notes offer a record of this anxiety:

Are studies sufficiently alike in their focus? Different decisions and different populations. Different sorts of literature - published and unpublished! (Memo, 15/05/12).

So for us, the act of reading was both a source of and a salve for anxiety. On the one hand it fuelled doubts about issues such as the suitability of selected accounts; on the other it assuaged concerns that 
the process would withstand scrutiny. Our meta-ethnographies have been conducted in an era when the authors of qualitative research are expected to demonstrate that their approach to data collection and to data analysis is thorough, systematic, and replicable. Being 'thorough' in many instances would mean being comprehensive, or even exhaustive, i.e. considering all available accounts. However, metaethnography was an approach devised in response to the challenges and possibilities associated with synthesising a small number of detailed monographs; Noblit and Hare (1988) demonstrated its use with two to six accounts. So whilst keen to practice meta-ethnography as originally conceived, we remained conscious throughout that this highly interpretive approach to synthesis, based on a small number of accounts, fitted somewhat uneasily with the prevailing tendencies in our field. As Campbell et al (2011) recognise, trying to maintain and manage a large dataset for the purposes of a meta-ethnography risks reducing the rigour of interpretative work and becoming reliant on procedural approaches to synthesis. Their solution was to group accounts in a medicine-taking synthesis by type of medication, but this practical step may not always be applicable to a meta-ethnography.

Our strategies for active reading provided reassuring evidence of systematics and attention to detail, a path towards the interpretive synthesis and a trail back to the contributing accounts. 'Active reading' was driven in part by the challenge of creativity and in part by the challenge of demonstrating credibility to our contemporaries. Critically it was underpinned by our coming together as a group, with the role of the collective being to provide essential challenge and support, both constraining and enabling our interpretations. On the one hand, the team provided a variety of checks and balances that work had been done, and could be evidenced, and that that interpretive leaps were plausible, i.e. checking any tendencies to 'read too much into things'. On the other, dialogue within the group helped 
us see different ways of engaging with and accessing the accounts, encouraged playfulness, and at key moments revitalised our reading.

\section{Searching for conceptual innovation}

Doing a meta-ethnography involves working with categories and metaphors detailed in the accounts of previous studies. Sometimes the categories are 'thin' - that is they are a description of an interpretive category, but are not supported by a discussion of how that interpretation was produced, or what the conceptual significance of the category named in the interpretation is. Sometimes they merely name the commonplace. Studies located within a particular field - dealing with young people's transition from child to adult health services, to use an example explored in this paper - can become fixed on using 'thin' categories and produce a certain genre of research ill-suited to the interpretive work comprising a meta-ethnography. The process of identifying studies to use in the meta-ethnography relies on search activities common to a variety of synthesis approaches, but settling on a group of suitable accounts may be frustrated by existing levels of conceptual development within a domain. In other words, the ingredients of the meta-ethnography are such that it is unlikely that conceptual development during the process of synthesis will be achieved. In such a scenario collaborative work is crucial in order to challenge on-going analysis, suggest alternative search questions and to recognise when conceptual innovation is not possible.

The investment in time and effort to engage in the repetitious and iterative interpretation of multiple accounts is considerable. Of course, in these circumstances it may become apparent that trying to undertake a meta-ethnography is futile, but the exploratory nature of meta-ethnography requires 
considerable investment before this judgment can be reached; the research team tries to make the meta-ethnography 'work'. Moreover, the ambition to deliver a meta-ethnography on a particular area of research or question can undermine the interpretive process and change the enterprise to one of aggregation. Associated with this aggregative imperative is an increase in the number of accounts used in order to justify the claims made for the synthesis.

Concern over conceptual innovation has been articulated most explicitly in meta-ethnography through reference to the ideas of Strike and Posner (1983) (see Britten et al, 2002; Campbell et al, 2003). Noblit and Hare (1988) drew on Strike and Posner to detail the criteria for judging a synthesis:

- clarifying and resolving inconsistencies and tensions;

- giving rise to a progressive problem shift;

- being consistent, parsimonious and elegant.

These criteria can also be thought of as indicators of conceptual innovation, though perhaps the last producing a consistent, parsimonious and elegant synthesis - is an ambition of all kinds of synthesis and research more generally.

Drawing upon two examples from our own work, it is possible to identify firstly, how particular methods of doing meta-ethnography are practiced in pursuit of these elements of conceptual innovation, and, secondly, how the best efforts of the meta-ethnographer may be frustrated by the nature of the accounts being synthesised.

Example 1: Testing and developing the concept of 'watchful restraint' 
$\mathrm{XW}$ sought to synthesise six accounts providing insights into the experiences of partners, family and friends of people with a musculoskeletal condition. These accounts were identified on the basis of ongoing literature searches. In engaging with the initial sample of papers, the research question was refined before a cohesive group was settled upon (this iterative selection of accounts is in-keeping with our earlier discussion). Efforts to achieve conceptual innovation were centred upon using coded extracts from the articles to begin to move from study-specific interpretations, through relations and translations, and towards the construction of caring practices helping to explain the original interpretations. To demonstrate how this process unfolded we can again focus on a particular example: the development of the caring practice of 'making space for watchful restraint'. The concept of watchful restraint was first discussed in a study by Mann and Dieppe (2006) in their analysis of illness management styles between couples. Originally located under a code of 'watching and anticipating', watchful restraint became the focus for collective discussion to think through the potential characteristics of the concept. The following is a note made by XW in reference to this discussion:

Restraint / holding back - suggestive of partners letting sufferer do their own thing, but also involves shaping and framing situation - telling things and moving away. Can therefore be a restraint on choice. Also, through delegation, as in the case of Hooper \& Ong, partner can limit the possibilities of sufferer by doing things for them - such as thinking and challenging (Memo: $13 / 06 / 12)$.

As a concept embedded in social processes, 'making space for watchful restraint' posed questions for the accounts included in the synthesis and required XW to make sense of the relationship between the emerging concept and the interpretations included in these accounts. The range of 
methods used to develop the concept included the use of open coding and axial coding as a step to developing a definition or narrative of the metaphor in question:

...after all the coding and things, I had this heading 'hybrid extracts and concepts', and we talked about this in a meeting, where I tried to start to write paragraphs which incorporated the metaphors. I was thinking about how to tell the story of how they fit across all the different studies....that was a bit more of a narrative...I suppose as a step towards thinking 'Are there any concepts that I can develop?' (Meeting note: 01/08/12).

In summary, making space for watchful restraint was developed through analysis of the accounts and then re-examining whether the resulting concept made sense when compared to those accounts. Importantly, these processes were undertaken on an individual and collective basis. In other words, watchful restraint was tested (or verified) by trying to understand how it helped to explain processes identified in other accounts, in a context of explaining and debating with team members. The concept, with an orientation towards the practices of carers, was thought to help explain the process by which carers of people with a musculoskeletal condition equip themselves with some knowledge about the condition, and an ability to act, remain vigilant, but try to hold back from intervening too quickly. Building on an existing category proposed by Mann and Dieppe (2006), the meta-ethnographic process involved testing and developing the concept through the interpretations of other relevant accounts.

Example 2: Being constrained by the policy narrative 
Giving rise to a progressive problem shift is difficult if the accounts to be used in a synthesis constrain the development of metaphors. $X Y$ conducted a meta-ethnography in the area of evaluations of transitional care, to inform on-going work. Existing literature discusses the various problems that may occur, potential reasons for these problems, and suggestions to smooth the transition from paediatric to adult care services (e.g. Dovey-Pearce and Christie, 2013). A previous review of models of transitional care found a tendency for pilot studies or new service developments to be described with limited evaluation or consideration of sustainability (Watson et al, 2011). In this respect producing a synthesis of existing service-change evaluations seemed an important contribution to understanding the state of the art. However, this meta-ethnography did not produce a synthesis which added any new insights to the field through conceptual innovation.

During the search phases of the meta-ethnography only a small number of qualitative studies evaluating service transitions were found, with only three deemed relevant. Judging whether these studies formed a 'coherent' collection - one that could provide insights amenable to a synthesis became a process of reading with a view to thinking about the end product (generating the kind of anxiety discussed in the previous section on reading). One of the papers, focused on a transition pathway in diabetes, proposed two super-ordinate themes: 'appropriate adolescent healthcare' and 'recognising individuality in healthcare'. It seemed - after much reading, re-reading, extracting of concepts/metaphors/chunks of text, and arranging and rearranging this information - that these two themes were replicated in all the articles on transition. Eventually, $X Y$ took this to a team meeting:

'XY: Well they fit into those two things. Also got "recognising individuality", in policy jargon. All these things fit in, [they] look at the individual. [They discuss] developmentally appropriate and 
individualised. Jargon. Ultimately, that is what all the papers argue ... Then in bed I started thinking have I done the right thing, because those [are] not essentially metaphors, they are policy things.

XZ: They are policy, they are policy ...

XY: And then I wondered had I been almost blindsided by policy.

XZ: Both [are] messages that policy wants to promote.

$\mathrm{XY}$ : Don't know whether these things doing because policy wants to promote, or whether promoting as that is what is coming out of the research' (Meeting note: 01/08/12).

The meaning and basis of the analysis presented in the accounts had become a problem for synthesis. In particular, a dilemma emerged about whether analysis embedded in policy terminology could offer a means of achieving conceptual innovation in the manner discussed by Strike and Posner (1983), Noblit and Hare (1988) and others. XY recognised that when looking at the service transition literature in general, these two themes - 'appropriate adolescent healthcare' and 'recognising individuality in healthcare' - are central to the whole canon of literature, while also fitting with the focus in UK health policy on 'person-centred planning'. Further, these themes overlap; if healthcare is individualised and person-centred planning used, then we will be undertaking developmentally appropriate healthcare, as it will be tailored to the individual. Continuing with the translation and synthesis of these studies seemed futile - despite collective attempts to share different readings of the accounts - with XY suggesting that:

... not sure whether to continue, almost a second attempt, or to accept that what I did before wasn't wrong, but just take that as the end point ... (Meeting note: 09/08/12). 
The 'fit' of concepts and metaphors in a meta-ethnography is a significant concern for those involved. In their account of conducting a second 'update' meta-ethnography in the area of participation in clinical trials, McCann et al (2013) discuss their concerns over the risk of forcing new studies to 'fit' their previous concepts. Whether considering how (or if) to rework a meta-ethnography, or revisiting a previously completed meta-ethnography, collaborative discussion is required about how best to proceed.

\section{Conclusions}

Our scrutiny of existing meta-ethnographies uncovered a variety of ways in which other individuals and teams have operationalised the seven core steps, or components, of meta-ethnography. This seemingly inconsistent approach to doing meta-ethnography provoked considerable debate within our team: 'Is there a "right" way to do meta-ethnography?' and 'What are the essential characteristics of metaethnography?' It was only through the conduct of, and reflection on, three distinct but concurrent metaethnographies of our own, that we were able to reach any consensus and to draw conclusions. We present these conclusions here in the hope that they may help colleagues make good decisions regarding 'whether' and 'how' to embark on a project of this sort.

\section{Meta-ethnography is a painstaking process, to which active reading is key}

Drilling down to the detail of our individual approaches in a way that is only possible through regular face-to-face interaction, it became clear that the variation we had observed in existing meta- 
ethnographies was mirrored in our own activities. Of course, we all made a case for our particular sequencing and choice of techniques being 'correct', in the sense of most in keeping with the spirit of meta-ethnography. However, over time we became convinced that it was not decisions to, for example, list or instead to tabulate which mattered, but the commitment - which we all shared - to intensive, repetitive and above all active reading. Active reading has varied and evolving manifestations (annotating, coding, listing and tabulating) and goals (to appraise, familiarise, extract, compare, etc.) Like good primary research it is painstaking and time-consuming; this is acknowledged by those who have gone before us (see for example Campbell et al., 2011). However, the lack of procedural detail in the majority of reports means that the labour inherent in this sort of approach has been far from clear. We hope that this paper, in focussing on the practical process, fills this gap.

\section{Meta-ethnography is an interpretive endeavour, through which multiplicity must be confronted}

Concepts and metaphors are the building blocks of the meta-ethnographic approach to qualitative synthesis. The use of metaphor implies an attempt, by the author(s) of an account, to articulate phenomena through the written word, and these articulations must then be interpreted by those conducting a meta-ethnography. Referencing the two-fold order of constructs (common sense and scientific thinking) set out by Schutz (1953), some authors (e.g. Britten et al, 2002) have referred to 'third-order' interpretations or constructs. We are not convinced that that the (re)interpretation of existing research accounts through synthesis gives rise to a new order of understanding. However, this is not to say that we consider a synthesis to be the same as an account of research; a synthesis has a different quality, arising from its objectives and the processes it involves. 
In reflecting on synthesis as interpretation, and the centrality of metaphor to this (whatever methods are deployed to try to fix and elucidate phenomena) we are reminded that in meta-ethnography one is always confronted with the other possibilities, other meanings. In this respect the question of allegorythe story of the synthesis - must be dealt with. For Law (2004: 97-98) allegory is 'apprehension of noncoherent multiplicity...or ways of knowing in tension.' Noblit and Hare (1988) themselves suggest that thinking through the value of allegory leads to the recognition that all is interpretation, and that the kind of synthesis produced by a meta-ethnography is open to re-interpretation and dispute. How then can one reconcile allegory - and the multiplicities this entails - with the search for a coherent and credible synthesis? This is a conundrum which underpins many of the procedural anxieties detailed in this paper. Unfortunately we can offer no easy answer here - it can only be addressed in context, i.e., in the process of actually doing a meta-ethnography and through dialogue with others.

\section{Meta-ethnography is not guaranteed to result in conceptual innovation}

We have already described in some detail how conceptual innovation may be constrained by the focus of the synthesis and the nature of relevant accounts. Attempts to retain difference - and restrain aggregation - while engaging with accounts (a concern of Noblit and Hare's) are influenced by substance and form of those accounts, which may offer restricted interpretations of the phenomena in question. Fleck (1935/1979) explains how the 'style-of-thought' or 'denkstil' shared by particular groups 'thought collectives' in Fleck's terms - can condition thinking and shape conceptual development within a field. So whilst the orientation of accounts to social processes allowed XW to identify and develop concept of 'watchful restraint', the health policy 'style-of-thought' underpinning the transitions literature left XY little room for interpretation and conceptual innovation. We learnt from this that not 
all accounts are suited to the meta-ethnographic approach, though this may not be immediately obvious. Making the decision to take the synthesis forward in another way, or not at all, is a hard one, due to the labour involved and the investment of time made before this point is reached. Again the team can play an important role in prompting an individual to confront such a decision, and inspiring, challenging, or validating the subsequent course of action.

\section{Meta-ethnography benefits significantly from being undertaken in collaboration with others}

Collaboration itself constrained, but also enabled the conduct of our meta-ethnographies. Working collectively drew our attention to the range of possible interpretations and invited us to question whether our personal interpretation might be intelligible and credible to a wider audience. Critically, the group validated $X Y^{\prime}$ 's conclusion that due to the nature of the relevant accounts, the endeavour in this particular instance should be abandoned.

Ultimately it seems to us that working as a collective encourages - and indeed requires - individuals to articulate their methods, evidence their engagement with the synthesised accounts, and to debate the implications of 'ingredients' and practices for the achievement of convincing conceptual innovation. This enhances the rigour of the process, the quality of the resulting synthesis, and - not inconsequentially - the meta-ethnographer's experience.

\section{Funding}


This research received no specific grant from any funding agency in the public, commercial or not-forprofit sectors.

\section{Acknowledgements}

The ideas presented in this paper were developed and refined over a series of presentations (Research Discussion Forum, Newcastle University, 4th September 2012; British Sociological Association Medical Sociology 44th Annual Conference, Leicester, 6th September 2012; British Sociological Association North East Medical Sociology Group Meeting, Northumbria University, 3rd October 2012). We would like to thank our audiences for their constructive comments and support.

\section{References}

Atkins S, Lewin S, Smith H, Engel M, Fretheim A and Volmink J (2008) Conducting a meta-ethnography of qualitative literature: lessons learnt. BMC Medical Research Methodology 8(21): 1-10.

Barry CA, Britten N, Barber N, Bradley C, Stevenson F (1999) Using reflexivity to optimize teamwork in qualitative research. Qualitative Health Research 9(1): 26-44.

Britten N, Campbell R, Pope C, Donovan J, Morgan M, Pill R (2002) Using meta ethnography to synthesise qualitative research: a worked example. Journal of Health Services Research \& Policy 7(4): 209-215. 
Cambrosio A, Keating P, Schlich T and Weisz G (2006) Regulatory Objectivity and the Generation and Management of Evidence in Medicine. Social Science \& Medicine 63: 189-199.

Campbell R, Pound P, Pope C, Britten N, Pill R, Morgan M and Donovan J (2003) Evaluating metaethnography: a synthesis of qualitative research on lay experiences of diabetes and diabetes care. Social Science and Medicine 56: 671-84.

Campbell R, Pound P, Morgan M, Daker-White G, Britten N, Pill R, Yardley L, Pope C and Donovan J (2011) Evaluating meta-ethnography: systematic analysis and synthesis of qualitative research. Health Technology Assessment 15(43).

Dey, I (1999) Grounding grounded theory: Guidelines for qualitative inquiry. Emerald Group Publishing Limited: Bingley.

Dixon-Woods M, Kirk D, Agarwal S, Annandale E, Arthur A, Harvey J, Hsu R, Katbamna S, Olsen R, Smith L, Riley R and Sutton A (2005) Vulnerable groups and access to health care: a critical interpretive review. Report for the National Co-ordinating Centre for NHS Service Delivery and Organisation R \& D (NCCSDO).

Dixon-Woods M, Cavers D, Agarwal S, Annandale E, Arthur A, Harvey J, Hsu R, Katbamna S, Olsen R, Smith L, Riley R and Sutton AJ (2006) Conducting a critical interpretive synthesis of the literature on access to healthcare by vulnerable groups. BMC Medical Research Methodology 6(35). 
Dovey-Pearce G and Christie D (2013) Transition in diabetes: young people move on - we should too. Paediatrics and Child Health 23(4): 174-179.

Doyle LH (2003) Synthesis through meta-ethnography: paradoxes, enhancements and possibilities. Qualitative Research 3(3): 321-44.

Fleck L (1935/1979) Genesis and Development of a Scientific Fact. Chicago: University of Chicago Press.

Flemming K (2010) Synthesis of quantitative and qualitative research: an example using Critical Interpretive Synthesis. Journal of Advanced Nursing 66(1): 201-17.

Geertz C (1973) The Interpretation of Cultures. New York: Basic Books.

Hammersley M (2013) The myth of research-based policy and practice. London: Sage.

Hannes K and Macaitis K (2012) A move to more systematic and transparent approaches in qualitative evidence synthesis: update on a review of published papers. Qualitative Research 12(4): 402442.

Hooper H and Ong BN (2005) When Harry met Barry, and other stories: A partner's influence on relationships in back pain care. Anthropology \& Medicine 12(1): 47-60. 
Law J (2004) After Method: Mess in Social Science Research. New York \& London: Routledge.

Mann C and Dieppe P (2006) Different patterns of illness-related interaction in couples coping with rheumatoid arthritis. Arthritis \& Rheumatism (Arthritis Care \& Research) 55(2): 279-86.

McCann S, Campbell M and Entwistle V (2013) Recruitment to clinical trials: a meta-ethnographic synthesis of studies of reasons for participation, Journal of Health Services Research \& Policy 18(4): 233-41.

Noblit GW and Hare RD (1988) Meta-ethnography: Synthesising Qualitative Studies. Newbury Park, CA: Sage.

Paterson BL (2012) 'It looks great but how do I know if it fits?': an introduction to meta-synthesis research. In Hannes K and Lockwood C (eds) Synthesizing Qualitative Research: Choosing the Right Approach. Chicester: John Wiley \& Sons.

Paterson BL, Thorne S and Dewis M (1998) Adapting to and managing diabetes. International Journal of Nursing Scholarship 30(1): 57-62.

Pound P, Britten N, Morgan M, Yardley L, Pope C, Daker-White G and Campbell R (2005) Resisting medicines: a synthesis of qualitative studies of medicine taking. Social Science \& Medicine 61: 133-55. 
Rice EH (2002) The collaboration process in professional development schools: results of a metaethnography, 1990-1998. Journal of Teacher Education 53(55): 55-67.

Sandelowski M, Docherty S and Emden C (1997) Qualitative Metasynthesis: Issues and Techniques. Research in Nursing \& Health 20: 365-371.

Schumm K, Skea Z, McKee L, and N'Dow J (2010) 'They're doing surgery on two people': a metaethnography of the influences on couple's treatment decision making for prostate cancer. Health Expectations 13: 335-349.

Schutz A (1945) On multiple realities. Philosophy and Phenomenological Research 5(4): 533-576.

Schutz A (1953) Common-sense and scientific interpretations of human action. Philosophy and Phenomenological Research 14(1): 1-38.

Soderberg S, Strand M, Haapala M and Lundmann B (2003) Living with a woman with fibromyalgia from the perspective of the husband. Journal of Advanced Nursing 42(2): 143-50.

Strike K and Posner G (1983) Types of syntheses and their criteria. In: Ward S and Reed L (eds) Knowledge structure and use. Philadelphia: Temple University Press. 
Thorne S, Jensen L, Kearney MH, Noblit G and Sandelowski M (2004) Qualitative Metasynthesis: Reflections on Methodological Orientation and Ideological Agenda. Qualitative Health Research 14(10): 1342-1365.

Tondeur J, van Braak J, Sang G, Voogt J, Fisser P and Ottenbreit-Leftwich A (2012) Preparing pre-service teachers to integrate technology in education: a synthesis of qualitative evidence. Computers \& Education 59: 134-44.

Watson R, Parr J, Joyce C, May C, LeCouteur A. (2011) Models of transitional care for young people with complex health needs: a scoping review. Child: Care, Health and Development 37(6): 780-791.

\section{Biographical notes}

